\title{
BLOCKCHAIN FOR ENERGY UTILITIES
}

\author{
KAFOL,C., BREgAR, A., TRILAR, J.
}

Abstract: Blockchain technology is disrupting the business landscape in many industries. Energy sector is conservative and has specific reliability and security requirements therefore the possible application range is relatively narrow. Authors research the benefits and shortcomings of the applied blockchain technology and suggest the possible areas in energy sector to develop the solutions based on blockchain principles. Usability of blockchain principles is under research and proper aim has to be defined in order to follow the energy utility goals. The aim od the article is to define the environment and methodology for application of blochchain principles for solving operational technology challenges at energy utilities. Authors also define the possible threats that do not enable fast technology adoption in power utilities biggest of which is cyber security.

Key words: blockchain, energy utilities, usability, methodology, cyber security
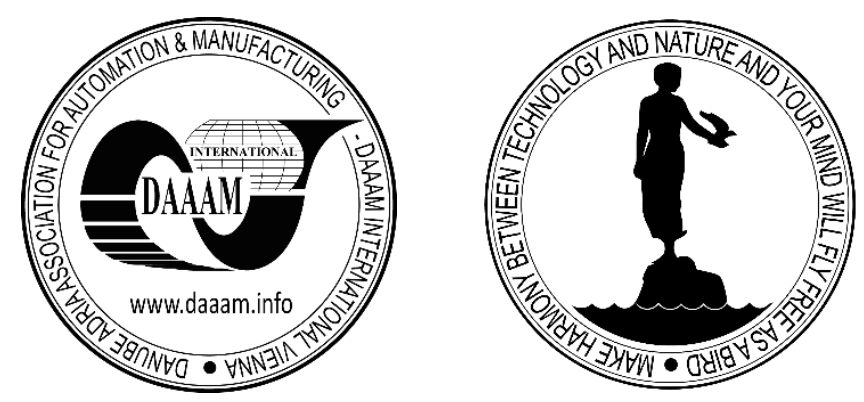

Authors' data: Dr Sc Kafol, C[iril]; Dr Sc Bregar, A[ndrej]; Trilar, J[ure], Informatika d.d., Vetrinjska ulica 2, 2000 Maribor, Slovenia, ciril.kafol@informatika.si

This Publication has to be referred as: Kafol, C[iril]; Bregar, A[ndrej] \& Trilar, J[ure] (2018). Blockchain for Energy Utilities, Chapter 15 in DAAAM International Scientific Book 2018, pp.159-174, B. Katalinic (Ed.), Published by DAAAM International, ISBN 978-3-902734-19-8, ISSN 1726-9687, Vienna, Austria DOI: $10.2507 /$ daaam.scibook.2018.15 


\section{Introduction}

Blockchain technology has wide use and is considered one of the disrupting technologies that will change the business landscape. There are many definitions of what the blockchain is and what its purpose is.

Wikipedia defines that the blockchain, originally »block chain «, is a growing list of records, called blocks, which are linked using cryptography. Each block contains a cryptographic hash of the previous block, a timestamp, and transaction data (generally represented as a Merkle tree root hash). By design, a blockchain is resistant to modification of the data. It is "an open, distributed ledger that can record transactions between two parties efficiently and in a verifiable and permanent way". For use as a distributed ledger, a blockchain is typically managed by a peer-to-peer network collectively adhering to a protocol for inter-node communication and validating new blocks. Once recorded, the data in any given block cannot be altered retroactively without alteration of all subsequent blocks, which requires consensus (Baliga, 2017), ussually of the network majority. Although blockchain records are not unalterable, blockchain may be considered secure by design and it exemplifies a distributed computing system with high Byzantine fault tolerance. Besides immutable records, various blockchains involve distinct innovative motivational mechanisms for agents that support data integrity of network infrastructure - enabling true decentralised consensus. Bitcoin as the first public showcase of blockchain was invented by Satoshi Nakamoto in 2008 to serve as the public transaction ledger of the first cryptocurrency. The invention of the blockchain for bitcoin made it the first digital currency to solve the double-spending problem without the need of a trusted authority or central server. The bitcoin design has inspired other applications, and blockchain platforms which are readable by the public are widely used by cryptocurrencies. Private blockchains have been proposed for business use. Some marketing of blockchain projects has been called "snake oil (Wikipedia, 2018).

Originally made for financial purposes or cryptocurrency several authors claim its wide usability and suggest several other uses. The blockchain is an incorruptible digital ledger of economic transactions that can be programmed to record not just financial transactions but virtually everything of value (Tapscott \& Tapscott, 2016).

According to 2017 Global benchmarking study (Hileman, Garrick and Rauchs, Michel, 2017) there are only 3\% of identified use cases in energy and utilities sector against 30\% in banking and finance sector and 13\% government and public goods sector. Sames study defines that potential target uses are $32 \%$ in energy market comparing to $70 \%$ in capital markets.

Authors will argue that there is larger potential of blockchain technology use in energy sector and therefore will define at least 3 use cases where the technology may be used with clear benefits for stakeholders in energy.

The research methods are inductive and deductive study of literature/trends and analysis (interview) within Slovenian energy sector stakeholders as well as market developments and projects. 
Authors will perform case study research combining the literature overview and trending thus providing theoretical framework and its comparison with practical application in the field. Combined approach should provide clear over-sight regarding usability of theoretical assumptions and define technology use.

\section{Maturity and usability of blockchain technology}

Uncertainty that is surrounding the use of technology is when will hundreds or perhaps thousands of pilots/demonstration projects come to production/usable phase. That is the grand question of technology maturity.

According to 2017 Global benchmarking study (Hileman, Garrick and Rauchs, Michel, 2017) $18 \%$ of platforms and services are still at the prototype stage. $39 \%$ of study participants promote platforms and services that are fully operational and productionready, and $36 \%$ are running advanced pilots (often with a beta access limited to a certain number of clients). This shows that activities are rather advanced, with threequarters of study participants having platforms that are live or nearly production-ready. However, sceptics or realists are suggesting (Frisby, 2018) that blockchain still has many shortcomings amongst which 3 stand out:

a) Blockchain cannot be everything it aspires to be at the same time.

As it stands, blockchain is caught between three competing objectives: fast, low-cost, and decentralized. It is not yet possible to make one chain that achieves all three. Fast and decentralized chains will incur a high cost due to the storage and bandwidth requirements for historical archiving will be enormous and will bloat even with pruning or other techniques.

b) Blockchain is a customer support nightmare.

For most consumers, losing a password to an online service is a mild inconvenience they've grown accustomed to, since typically, it's quickly fixed by requesting an email reset, say, or talking with customer service. Blockchain wallets and their passwords, by contrast, are tied to a file on a user's hard disk and are absolutely critical to users trying to access the blockchain. By their very nature they have no recovery mechanism. "You lose your password, you lose everything" is an awful user experience for mainstream consumers and a nightmare for companies attempting to build their service on a blockchain. If you use a hosted service, the risk of theft or sudden loss of assets is very real, with central targets and limited traceability.

c) Blockchain adds frictions to an already seamless process.

Nothing about blockchain applications is easy for consumers right now. Everyday users accustomed to making digital and online payments would have to be trained to make blockchain purchases, learning to apply the right mix of paranoia and caveat emptor to prevent theft or buying from shady dealers. Irreversible pseudonymous transactions do not lend themselves well to trust and integrity. Compounding this is the speed and the transaction fees involved. Most public chains have settlements measured in minutes unless you're willing to pay high transaction fees. Compare

that to the 2-10 seconds for a saved credit card transaction customers are accustomed to in the age of fast mobile interfaces and instant gratification. We have identified 2 additional significant risks that involve (Marr, 2018): 
d) Lack of regulation and control creates uncertainty in business environment. Legislative and governmental bodies are forced to take action against individual platforms and blocking applications due to cyber-threats or business models that are inconsistent with the regulations (Aune, 2018). New business models and nature of these transaction are under scrutiny of administrations in most of the developed countries, though clear operative instructions for legislators and controlling bodies are scarce.

e) Complexity of the technology generates widespread assumption that this technology is not usable enough.

The concepts of encryption, decentralization and distributed consensus that are the basis of block technology are often too complex to understand for average citizen. Models of distributed transactions and smart contracts that eliminate intermediary roles such as in financial institutions or suppliers in the energy market and other benefits are not recognised. Consequently, the potentials of the usefulness of technology are not perceived as viable and useful in the eye of the public. Without sufficient adoption and public support of the technology, it is difficult to justify the need for blockchain in long-term.

It is necessary to consider the fundamental problems that we are trying to solve with this technology - as some restrictions, that do not necessary originate in technical approaches towards the design of blockchain, can be vital for success or fail in terms of widespread use (commercialisation) among public. So far, we can only assess that the blockchain technology is not an universal answer to all the problems.

\section{Defining the use of blockchain in energy utilities}

Energy or electric utility is a company in the electric power industry (often a public utility) that engages in electricity generation and distribution of electricity for sale generally in a regulated market. (Wikipedia, 2018). Energy utility has to be conservative in its approach to adopting new technologies as its operation is vital to supply of electric energy to retail and wholesale consumers. Therefore we will try to find use cases in areas not directly influencing the operations of utilities or delivery of energy.

Gartner defines blockchain as trend no.8 within 10 Gartner strategic technology trends for 2018 (Gartner, 2018) and also digitalization as one of the main trends in energy utilities sector in article Transforming Into a Digital Utility Primer for 2018 (Gartner, 2018). However, we are still at the beginning of the digitalization process in utilities and it may take longer time to adopt new technologies. Nonetheless, digitalisation of processes is bringing new treats into energy utility operations, biggest of which is cyber security. Energy sector is the second most attractive sector for cyberattacks, which is shown by the number of attacks, while finance sector is first.

We have yet to see the emergence of dominant networks with a considerable number of participants that have established themselves as platforms upon which applications can be built. 
For this reason, the number of publicly known applications built on enterprise distributed ledger networks is still rather small, and the majority constitute permissioned applications that are built on the public Bitcoin or Ethereum mainnets (Hileman, Garrick and Rauchs, Michel, 2017).

\section{Defining the use cases for energy utilities}

Various applications and use cases of blockchain technologies are emerging in different business domains (Madhwal \& Panfilov, 2017; Rampton, 2018), including the utilities sector (PwC, 2016). Despite the assumed technological and conceptual suitability of blockchain, many of its potentials have not been sufficiently utilized yet. The authors argue that it is possible to apply blockchain mechanisms in several use case scenarios in relation to both regulated processes as well as (partially) nonregulated business processes in the utilities sector. These scenarios are summarized in Table 1.

\begin{tabular}{|l|l|l|l|l|}
\hline Use case & Access & Participation & Domain & Model \\
\hline $\begin{array}{l}\text { Exchange of data among energy } \\
\text { stakeholders for operating energy } \\
\text { market }\end{array}$ & $\begin{array}{l}\text { Permissioned, } \\
\text { permissionless }\end{array}$ & Collaborative & Regulated & $\begin{array}{l}\text { Contract, } \\
\text { ownership }\end{array}$ \\
\hline $\begin{array}{l}\text { Registrations of the ownership of } \\
\text { energy resources, such as } \\
\text { measurement devices and energy } \\
\text { generation devices }\end{array}$ & $\begin{array}{l}\text { Permissioned, } \\
\text { permissionless }\end{array}$ & Collaborative & Regulated & Ownership \\
\hline $\begin{array}{l}\text { Certifications and guarantees for } \\
\text { renewable energy resources and } \\
\text { emissions }\end{array}$ & $\begin{array}{l}\text { Permissioned, } \\
\text { permissionless }\end{array}$ & Collaborative & Regulated & Ownership \\
\hline $\begin{array}{l}\text { Smart contracting for the } \\
\text { distributed energy producers }\end{array}$ & $\begin{array}{l}\text { Permissioned, } \\
\text { permissionless }\end{array}$ & $\begin{array}{l}\text { Collaborative, } \\
\text { competitive }\end{array}$ & $\begin{array}{l}\text { Regulated, } \\
\text { business }\end{array}$ & Transaction \\
\hline $\begin{array}{l}\text { Management and optimization of } \\
\text { energy networks based on } \\
\text { demand and supply }\end{array}$ & $\begin{array}{l}\text { Permissioned, } \\
\text { permissionless }\end{array}$ & $\begin{array}{l}\text { Collaborative, } \\
\text { competitive }\end{array}$ & $\begin{array}{l}\text { Regulated, } \\
\text { business }\end{array}$ & $\begin{array}{l}\text { Contract, } \\
\text { transaction }\end{array}$ \\
\hline $\begin{array}{l}\text { Introduction of cryptocurrency } \\
\text { for internal energy trading within } \\
\text { closed energy ecosystem }\end{array}$ & Permissionless & $\begin{array}{l}\text { Collaborative, } \\
\text { competitive }\end{array}$ & Business & $\begin{array}{l}\text { Contract, } \\
\text { transaction }\end{array}$ \\
\hline $\begin{array}{l}\text { Energy measurement and billing } \\
\text { Billing of energy for e-mobility }\end{array}$ & Permissionless & $\begin{array}{l}\text { Collaborative, } \\
\text { competitive }\end{array}$ & Business & $\begin{array}{l}\text { Contract, } \\
\text { transaction }\end{array}$ \\
\hline Communication of smart devices & Permissionless & $\begin{array}{l}\text { Collaborative, } \\
\text { competitive }\end{array}$ & Business & Transaction \\
\hline
\end{tabular}

Tab. 1. Blockchain use cases for energy utilities

Scenarios exhibit different complexities. It is relatively straightforward to manage the ownership of data, documents, certificates and devices. Many innovative approaches, however, appear when we introduce competitive and collaborative models among electricity suppliers and distributors, direct storage of measurement data in blockchains, or public permissionless models in which energy producers and consumers can obtain security certificates from the central authorities and hence set up 
their own blockchain nodes. In this way, a decentralised energy market can be formed that allows prosumers, i.e. entities which are producers and consumers of electricity at the same time, to directly sell energy to consumers independently of intermediary suppliers. Criptocurrencies represent a key asset in these scenarios.

Based on the current state and maturity of the blockchain technology, some of the identified use cases are not applicable, or are applicable only partially within considerable constraints. The main restrictions pertain to the scalability and transaction speed. Moreover, some scenarios require regulatory changes, which will be difficult to introduce and implement, although they have been to some extent initiated by regulatory authorities and standardization organisations, among which are the Slovenian Energy Agency and the ebIX forum (Energy Agency, 2017). These changes have to consider the regulated processes, role models and data structures in the utilities sector.

According to the above limitations, the literature study and interviews with stakeholders in the Slovenian power distribution network, authors have cleared out three key use cases of blockchain considering the impacts and trends in the industry. By comparing this and following interviews with the stakeholders in the industry authors are suggesting the possible uses as follows:

1) Introduction of cryptocurrency for internal energy trading within the closed energy ecosystem

2) Smart decentralised contracting for distributed energy producers

3) Exchange of data among energy stakeholders for operating energy market

\section{Use case 1: Introduction of cryptocurrency for internal energy trading within the closed energy ecosystem}

The closed energy ecosystem or the microgrid represents a great opportunity to apply blockchain technologies. It results in self sufficient smart communities with energy generation devices based on renewable energy sources. Local producers are able to directly sell energy to neighbouring consumers, without the involvement of intermediary suppliers which operate on the national or regional level. As a consequence, prices can be determined dynamically according to the demand and supply in the microgrid. Moreover, the process of the change of supplier does not need to be implemented and regulated.

In this scenario, blockchain securely persists transactions on the production and supply of electricity. It also allows producers and consumers to get directly involved into smart contracts. The foundations of such a system are as follows (PwC, 2016):

- Smart contracts effectively balance the supply and demand, and are hence able to control electricity grids with regard to the energy production, consumption and storage. Such active balancing can significantly improve energy efficiency, especially in sustainable environments. Several studies have shown that energy efficiency of electricity grids is a particularly important topic (Micieta et al., 2015; Rogobete et al., 2015; Teich et al., 2017).

- Transaction data is persisted in a secure decentralised blockchain that can be accessed by the contracting parties through their digital identities. 
- Transactions are executed autonomously according to the terms of smart contracts. They influence the amount of energy that is supplied through the physical electricty network.

- Consumers pay for blockchain transactions by the means of a criptocurrency. No traditional billing systems are required.

- Blockchain also manages various ownerships by storing and processing data on the ownerships of production devices, certificates, etc.

- Physical and applicative infrastructure of blockchain based energy trading systems must incorporate smart electrical devices, smart meters, sensors, etc.

The first application of a decentralised blockchain system for internal energy trading has already been implemented (Mearian, 2018). It is a microgrid of a small self sufficient smart community in Brooklyn in New York. It operates independently of the central regional electricity network. It is therefore fully and autonomously responsible for all of its production and consumption of electrical energy that comes from renewable sources. All transaction data is stored in an Ethereum blockchain. Cryptocurrency is the basic payment method.

\section{Use case 2: Smart decentralised contracting for distributed energy producers}

According to the current state of technology, the first defined use case is suitable only for smaller closed ecosystems. The key restriction is the scalability of blockchain, which is insufficient for large electricity distribution systems that have to store and process millions of transactions on energy consumption. These opened and regulated systems can, however, gain substantial benefits from smart decentralised contracting between permitted parties. Smart contracts are one of the most important inherent concepts of blockchain (Bartoletti \& Pompianu, 2017; Blockgeeks, 2018).

In this use case, energy producers sign contracts with multiple parties. Energy consumers take the role of the contracting parties on one side, while on the other side, smart contracts must be signed and approved by major entities in the utilities system, such as distributors, suppliers, regulators and data aggregators. The latter entities are also the owners and administrators of blockchain nodes. They are entitled the rights and responsibilities to implement the signed terms of contracts. This means that the produced energy is indirectly supplied to consumers by electricity suppliers over the standard distribution grid, and that data on measurements is obtained, aggregated and supervised by national distribution operators and regulators. Billing and data exchange are also independent of the blockchain technology, and are performed with standard transaction and integration based systems.

The main benefit of blockchain in this use case is that contracts are made dynamically based on the demand and supply. This enables producers and suppliers to actively optimize their offers and services, and consumers to actively identify and choose the best energy providers. In addition, the regulated processes of formulating, signing and following formal contracts between many interdependent parties become substantially more transparent, automated, secure, and easy to manage. 
Each party is not required to directly communicate with all others, and locally store its own copies of documents. Instead, the entire processes are executed in the decentralised front-end of blockchain, and securely managed in the back-end of blockchain.

\section{Use case 3: Exchange of data among energy stakeholders for operating energy market}

The exchange of data in the energy sector is frequent and well defined (ebIX, 2018). Aggregated data is generally exchanged among different roles, which include distributors, suppliers, system operators and regulators. This use case should be combined with the previous use case. At first, a private agreement in the form of a smart contract is made among the participating parties to formally determine which roles exchange data, what data is exchanged, and under which conditions. Afterwards, data is securely exchanged, possibly on a recurring basis. There are two available approaches to exchange data. The first possibility is that one entity writes data directly into blockchain from where the receiving entity reads it. However, because aggregated data is in most scenarios intended for only one entity, it is usually unnecessary to store it in a distributed manner in several encrypted nodes. The exchange is therefore more likely to be executed indirectly with the service-oriented architecture (SOA) (Erl, 2009) based integration that utilizes regulated data exchange processes and standardised data structures as proposed by several authorities (ebIX, 2018; Energy Agency, 2017). Secure data services or message queues are applied for this purpose, while contractual data from blockchain nodes is used only to establish safe communication paths.

\section{Prototype blockchain solution for energy utilities}

We have developed a prototype blockchain solution for energy utilities that follows use cases 2 and 3. We have defined the blockchain scenario and architecture in two workshops. The first workshop has been attended by a large number of domain experts. We have conducted structured interviews and used several idea generation techniques (Cleverism, 2015; Herring et al., 2009) in order to identify the scenario and its main characteristics, analyse the requirements and expectations of the energy distribution system, and assess the capabilities of available blockchain technologies. We have considered various criteria:

- the EU General Data Protection Regulation (GDPR) (European Commision, 2018), which is crucial to restrict the prototype solution to domain data that is not critical with regard to the legal security;

- prevention of process and data manipulations, unauthorised access to business policies and data, and cybernetic risks;

- efficient and meaningful alignment of the prototype with existing processes in the utility domain;

- direct usability within the scope of existing information systems developed to support processes in the utility domain; 
- technical feasibility with regard to the amount of data required for storage and processing, transaction amount, transaction speed, and the quantity of required blockchain nodes;

- ability of rapid and easy development and prototyping;

- availability of a mature public platform that would not impose development risks and security threats;

- suitability to clearly demonstrate the technological and conceptual abilities and benefits of the chosen architecture and use cases within a wider environment.

After the first workshop, we have formally evaluated several available blockchain platforms: Bitcoin, Ethereum, Hyperledger, Neo (former Antshares), Iota, Eos and Ada Cardano. Numerous criteria, such as the existence of cryptocurrency in a direct correlation with the platform, availability of smart contracts, type of consensus, speed and costs of transactions, type of network, degree of decentralisation, access, anonymity, suitability for IoT, operational costs, state channels, community support, etc., were considered. After a lengthy analysis, the Ethereum smart contract platform (Ethereum, 2018) was chosen as the most suitable platform for the purpose of our use case. Fundamental features of the Ethereum platform, that enabled us with enough certainty that a functional prototype could be implemented, were:

- rapid acquisition of data from the Ethereum platform, which generally does not exceed 300 milliseconds;

- lower write delay and record validity check, which typically requires block generation time of 3 to 5 minutes;

- more constant generation of blocks compared to the Bitcoin network;

- various development environments and libraries, such as Truffle, Remix Web IDE etc .;

- software logic processing in the running environment of Ethereum Virtual Machine (Prusty, 2017), which enables high decentralization provided with 27,500 hubs around the globe;

- the development of smart contracts in the solitary language Solidity (Prusty, 2017);

- integration with advanced web applications based on the Ethereum JavaScript application programming interface (Web3.js) (Ethereum Javascript API, 2018), which represents a potential to a wide range of available user scenarios;

- an indigenous means for transactions fees in the form of a cryptocurrency Ether in the "aggregate state" of Gas (Cryptocompare, 2018), which represents the micro-units of the primary currency. In praxis, transaction costs are lower than commission for Bitcoin transactions;

- future proof concepts implementations, such as eventual transformation from the energy-wasting PoW (Proof-of-Work) protocol to the PoS (Proof-of-Stake) protocol;

- development possible in various development-testing networks (Ropsten, Rinkeby, Kovan or RPC Custom) in which Ether is not consumed for testing purposes as on the mainnet; 
- the largest consortium of industry supporters (Enterprise Ethereum Alliance).

On the second workshop, we have used the card sorting technique (Spencer, 2009) to define the set of key concepts and functionalities of the prototype, detailed system architecture, data structures, and participating roles in the data exchange process, as well as the content, clauses and rules of the smart contract, enclosed in meaningful user scenarios (Axup, 2018). As a result, we have developed and introduced a blockchain solution that, in compliance with the regulatory requirements, grants electricity suppliers an exclusive indirect access to the measurement data that is obtained for consumers and producers, and is owned by electricity distribution companies. Several scenarios to access the measurement data by invoking data exchange services are available under the authority of the advanced measurement centre (Energy Agency, 2017). In the implemented prototype solution, a subset of selected scenarios is supported. They are formalised by the clauses of the smart contract on the use of data services that allow the access to the measurement data of an individual electricity consumer/producer. The clauses are as follows:

1. clause: The supplier of electrical energy is granted web access to 15-minutes based measurement and billing data for the metering point that belongs to the hereby signed electricity consumer or producer. Data will be provided on the monthly basis for the period of the last 12 months.

2. clause: The supplier of electrical energy is granted web access to 15-minutes based measurement data for the metering point that belongs to the hereby signed electricity consumer or producer. Data will be provided for the last month only.

3. clause: The supplier of electrical energy is granted web access to aggregated statistical measurement and billing data for the metering point that belongs to the hereby signed electricity consumer or producer. Data will be provided for the period of the last 24 months.

The measurement data on the consumption of electricity belongs to individual clients, and is owned by distributors. The access to this data is consequently restricted for suppliers, however it is valuable for them in order to develop optimal services and strategies, and remain competitive on the market. Our blockchain application allows a client to indirectly, through the distributor's blockchain front-end, grant his supplier an insight into his personal measurement data, whereby regulatory restrictions are strictly met. In this way, the supplier becomes able to analyse a set of 15-minutes based consumption measurements, and can work on an optimal business offer that is specifically tailored to the individual. With the mutually signed smart contract, rules are set that are acceptable for all parties.

The creator of the contract is either the supplier or the distributor. End clients cannot set up their own blockchain nodes, but rather use their public keys as identities to access the nodes of suppliers or distributors through the secure web user interface. When the client signs one or more clauses of the smart contract with his private key, a new transaction is created in the block. This transaction is non-modifiable, and is permanently readable for both the client that has created it as well as the node owner (administrator). 
The transaction data identifies the metering point, energy distributor, energy supplier, and the period for which the measurement data is obtained. The supplier receives the granted measurement data from the distributor by the means of serviceoriented integration.

The prototype consists of the back-end and the front-end. The back-end is based on the smart contract, which specifies the main application logic that defines how the data on users and contract clauses is written in array structures, and is published on the Ethereum blockchain. The front-end enables a user friendly interaction with the Ethereum blockchain. See Figure 1 for a graphic view and detailed layout description. The developed program code is freely available on the GitHub portal. Most of the established design patterns for decentralised apps are applied (Bartoletti \& Pompianu, 2017; Bontje, 2015). This approach has captured all the practical aspects of current blockchain technology in the form of decentralised application:

- tokenisation and transactions,

- identity management,

- smart contracts written in smart contracts.

Decentralised applications (dApp) typically include common traits that processing and backend transactions take place on a decentralized peer-to-peer network, as opposed to applications where background data is stored and processed on centralized servers. DApps existed before the invention and popularization of blockchain, practically since the beginning of early P2P networks. This type of software was designed to exist on the Internet in such a way that it works completely autonomously and can not be controlled from the side of one entity. Most dApps are based on web platforms, which are run in web browsers - similar to classic websites and common web applications. Further, client interface resource can be hosted on decentralised hosting, such as Swarm or IPFS (Interplanetary file system), to improve data retention and robustness. From all the available sources, a definition of four basic features of modern dApps is drawn:

- autonomy and openness,

- using blockchain for storing data or database,

- use of cryptographic tokens to store values or assets,

- the main operational logic is written in smart contracts.

Figure 1 represents screenshots of a demo made under demonstration project. Its purpose is to show usability of blockchain principles in real projects. Left part of picture is a smart contract web interface that is publicly available to consumer; access and signing of distinct clauses is authenticated via Metamask browser plugin. Right part of picture represents administration dashboard, intended for access control, utility token distribution, addition of clauses, and viewing lists of signatures under particular clause. 
Kafol, C.; Bregar, A. \& Trilar, J.: Blockchain for Energy Utilities

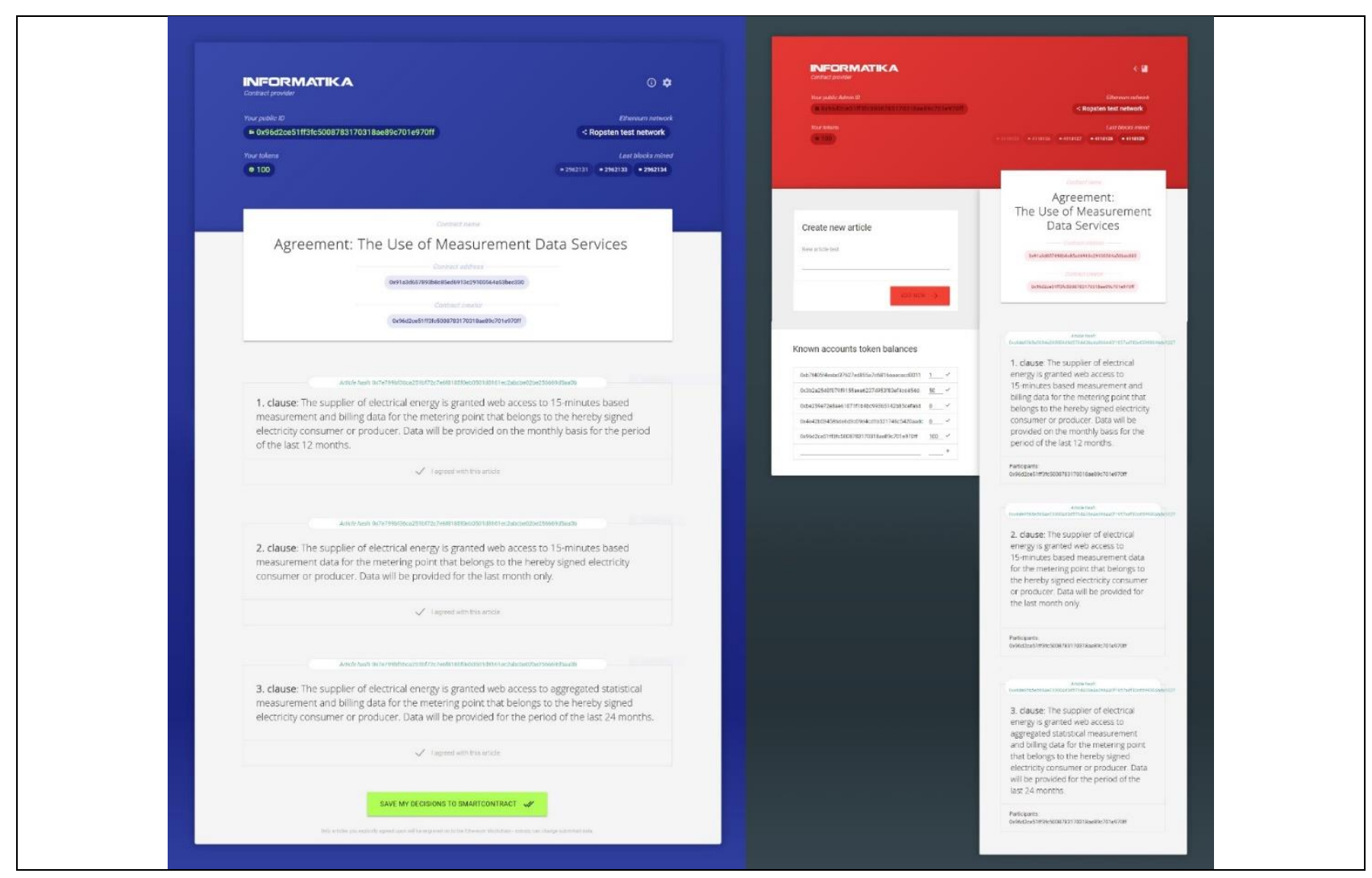

Fig. 1. Application client public and administration front end

\section{Cyber security issues}

The Ethereum platform has proven to be effective and useful to quickly and easily develop a prototype solution for the selected use cases in the energy utility domain. However, we could face many obstacles if we would choose to implement some of the more complex use cases from Section 4, especially because the blockchain technology is still in an early development phase, and has therefore many weaknesses that are known to developers, but are regardless of this fact often overlooked or insufficiently considered when designing applications and solving problems. Many of these drawbacks are related to cyber security issues, for example:

- total loss of data in the case of identity loss;

- cybernetic risks because of potential attacks on the network level, both in the computer networks as well as in the operations networks, for example in the case of manipulating smart metering devices;

- security risks related to low scalability, insufficient network flexibility and performance issues;

- security risks due to insufficient standardisation and regulation;

- security risks because of inappropriate or restrictive regulative requirements for some use cases;

- security risks because of weak technological suitability for some use cases;

- security vulnerabilities due to potential weaknesses in the architectural design because of technology immaturity, lack of development experience, and poor documentation and support; 
- security vulnerabilities due to potential weaknesses in the functional design because the technology is prone to low user acceptability as a consequence of its complexity, incomprehension and mistrust;

- lack of a central regulatory entity that would resolve legal disputes.

Marr (2018) states that the lack of regulations and control establishes a risky environment which is considerably prone to cyberattacks. This environment is exposed to significant opportunities for such threats. It is vital to understand, that with newly emerged direct financial gains for a hacker in the form of cryptocurrencies, almost all the world's hacking power is targeted at blockchain - most of the cases in a non-direct manner, though minority of these attacks are directed at exploits in different blockchain platforms. Numerous cases of attacks, hacks and abuses are documented, often resulting in substantial financial losses. It can also happen that governmental institutions and regulatory bodies are forced to take measures against blockchain platforms and applications because of cyber security risks.

Cyber Defense Magazine (2018) reports on many serious incidents that pertain to blockchain technologies and cryptocurrencies. They are linked to Bitcoin, Tether, Nicehash and many others cases. Million of dollars of losses are also reported by Information Security Media Group (2016) and Coindesk (2017). Some blockchain experts have therefore proposed different recommendations on how to avoid vulnerabilities to cyber security attacks, and to protect blockchain based systems from hacks (CSO, 2017). A few blockchain solutions also exist that claim to have overcome vulnerabilities to attacks, and that are able to protect user data from being modified (Wolfson, 2018).

\section{Conclusion}

Blockchain is becoming an increasingly popular and widespread technology, but its potentials have not been fully realised in the energy utilities sector yet. For this reason, we have conducted a thorough research on the state-of-the-art, possibilities, opportunities and pitfalls of blockchain in this sector. The study has incorporated several research methods, including the literature review, interviews with domain experts, the use of idea generation and structuring techniques, comparative analyses, prototyping and case based experiments. As a result, we have identified and proposed key use cases, provided recommendations on how to efficiently apply the blockchain technology and how to avoid its weaknesses, assessed the most relevant platforms and selected the one that is sufficiently suitable for the energy utilities domain, as well as developed a fully operational prototype to support the regulated processes of data exchange in the electricity market. We have also evaluated this prototype by the means of a case based study.

We have shown that blockchain can be efficiently used, however we have to be aware of its shortcomings, which are a consequence of the fact that blockchain is still evolving and has not become a fully mature technology yet. We have hence determined that not all potential use case scenarios are currently appropriate to be implemented. The future research will address these particular scenarios. 
We will try to advance the blockchain technology and methodology by studying, developing and introducing innovative solutions that will overcome known pitfalls and consequently enable a less restrictive, wider, safer and even more efficient use of blockchain based applications.

In order to introduce blockchain technology based solutions with a higher innovative potential and general usefulness, it is necessary to overcome the shortcomings and limitations at both ends, the technology level, particularly in terms of maturity and scalability, as well as at the regulatory and business level, in regard to the public acceptance of this technology. If it turns out that the problems are primarily that of technological nature and the different blockchain platforms are conceptually appropriate for wider use, there may enough space for significant technological advancements in several economic sectors.

\section{References}

Aune, R. T.; Krellenstein, A.; O’Hara, M., \& Slama, O. (2017). Footprints on a Blockchain: Trading and Information Leakage in Distributed Ledgers. The Journal of Trading,12(3), 5-13. doi:10.3905/jot.2017.12.3.005

Axup, J. (2018). UX for Cryptocurrencies - The Revolution Won't Happen Without It, Available from: https://medium.com/@jeffaxup/ux-for-cryptocurrencies-therevolution-wont-happen-without-it-part-1-of-3-a896855dfe82 Accessed; 2018-04-08 Baliga, A. (2017). Understanding Blockchain Consensus Models, Persistent Systems, White Paper, Available from: https://www.persistent.com/wpcontent/uploads/2018/02/wp-understanding-blockchain-consensus-models.pdf

Accessed:

2018-06-22

Bartoletti, M. \& Pompianu, L. (2017). An Empirical Analysis of Smart Contracts: Platforms, Applications, and Design Patterns. Financial Cryptography and Data Security Lecture Notes in Computer Science,494-509. doi:10.1007/978-3-319-702780_31

Blockgeeks (2018). Smart Contracts: The Blockchain Technology That Will Replace Lawyers, Available from: https://blockgeeks.com/guides/smart-contracts/_Accessed: 2018-05-26

Bontje, J. (2015). DApp design patterns: Emerging best practices in the world of Decentralized Applications, Available from: www.slideshare.net/mids106/dappdesign-patterns Accessed: 2018-06-30

Cleverism (2015). 18 Best Idea Generation Techniques, Available from: https://www.cleverism.com/18-best-idea-generation-techniques/ Accessed: 2017-1022

Cryptocompare (2018). What is the »Gas « in Ethereum? Available from: www.cryptocompare.com/coins/guides/what-is-the-gas-in-ethereum/ Accessed: 2018$09-22$ 
CSO (2017). Hacking bitcoin and blockchain, Available from: https://www.csoonline.com/article/3241121/cyber-attacks-espionage/hackingbitcoin-and-blockchain.html Accessed on: 2018-03-23

Cyber Defense Magazine (2018). Bitcoin, BlockChain and Breaches, Available from: http://www.cyberdefensemagazine.com/bitcoin-blockchain-and-breaches/_Accessed: 2018-07-20

De, N. (2017). Hacks, Scams and Attacks: Blockchain's 2017 Disasters, Available from: https://www.coindesk.com/hacks-scams-attacks-blockchains-biggest-2017disasters/ Accessed: 2018-03-20

ebIX (2018). European forum for energy business Information eXchange, Available from: https://www.ebix.org/ Accessed: 2018-08-09

Slovenian Energy Agency (2017). Regulative changes to establish a new prosumer role in the energy market, Available from: www.agenrs.si/documents/10926/106759/Regulativne-spremembe-za-vzpostavitev-nove-vlogena-trgu-Aktivni-odjemalec/6a00e54d-e9c8-419f-99a4-e7e2dcf0c3b9 Accessed: 2017$12-15$

Erl, T. (2009). Service-Oriented Architecture: Concepts, Technology, and Design, Prentice Hall, Upper Saddle River, New Jersey

Ethereum Foundation (2018). Ethereum JavaScript API, Available from: http://github.com/ethereum/web3.js Accessed: 2018-05-13

Ethereum Foundation (2018). Ethereum, Available from: http://www.ethereum.org, Accessed on: 2018-07-03

European Commision (2018). Data protection in the EU, Available from: https:/ /ec.europa.eu/info/law/law-topic/data-protection/data-protection-eu_en Accessed: 2018-07-120

Frisby, A. (2018). Why blockchain isnt't ready for the primetime. Sinespace. Available from: https://venturebeatcom.cdn.ampproject.org/c/s/venturebeat.com/2018/03/11/why-blockchain-isnt-readyfor-primetime/amp/ Accessed: 2018-07-06

Gartner (2018). Energy and Utilities in the Digital Age: 10 trends, Available from: https://www.gartner.com/en/industries/energy-utilities Accessed: 2018-06-01

Gartner (2018). Transforming Into a Digital Utility Primer for 2018, Available from: https://www.gartner.com/doc/3844864 Accessed: 2018-03-10

Herring, S. R.; Jones, B. R. \& Bailey B. P. (2009). Idea Generation Techniques among Creative Professionals, 42nd Hawaii International Conference on System Sciences, https://doi.org/10.1109/HICSS.2009.241

Hileman, G.; Rauchs, M. (2017). Global Blockchain Benchmarking Study, Available at SSRN: https://ssrn.com/abstract=3040224 or http://dx.doi.org/10.2139/ssrn.3040224

Information Security Media Group (2016). Bitcoin Hack Highlights Cryptocurrency Challenges, Available from: https://www.bankinfosecurity.com/bitcoin-hackhighlights-cryptocurrency-challenges-a-9305 Accessed: 2018-04-25

Madhwal, Y. \& Panfilov, P. (2017). Blockchain And Supply Chain Management: Aircrafts' Parts' Business Case, Proceedings of the 28th DAAAM International 
Symposium, pp.1051-1056, B. Katalinic (Ed.), Published by DAAAM International, ISBN 978-3-902734-11-2, ISSN 1726-9679, Vienna, Austria

Marr, B. (2018). The 5 big problems with blockchain everyone should be aware of, Forbes, Available from: www.forbes.com/sites/bernardmarr/2018/02/19/the-5-bigproblems-with-blockchain-everyone-should-be-aware-of/\#5adf0c661670 Accessed: 2018-08-28

Mearian, L. (2018). 5 ways blockchain is the new business collaboration tool, Computerworld, Available from: www.computerworld.com/article/3197695/blockchain/5-ways-blockchain-is-thenew-business-collaboration-tool.html Accessed: 2018-08-23

Micieta, B.; Zavodska, L.; Rakyta, M. \& Binasova, V. (2015). Sustainable Concept for Green Logistics and Energy Efficiency in Manufacturing, Chapter 33 in DAAAM International Scientific Book 2015, pp.391-400, B. Katalinic (Ed.), Published by DAAAM International, ISBN 978-3-902734-05-1, ISSN 1726-9687, Vienna, Austria Prusty, N. (2017). Building Blockchain Projects: Building Decentralized Blockchain Applications with Ethereum and Solidity, Pack Publishing, Birmingham

PwC Global Power\&Utilities (2016). Blockchain - an opportunity for energy producers and consumers? Available from: www.pwc.com/gx/en/industries/energyutilities-resources/publications/opportunity-for-energy-producers.html Accessed: 2018-08-10

Rampton, J. (2018). 5 applications for blockchain in your business, TheEconomist Careers Network, Available from: https://execed.economist.com/blog/industrytrends/5-applications-blockchain-your-business Accessed: 2018-08-05

Rogobete, M.; Pintilie, I. \& Scutaru, M. (2015). A Means of Allocating the MW Requirement in an Electrical Power System, Chapter 25 in DAAAM International Scientific Book 2015, pp.299-310, B. Katalinic (Ed.), Published by DAAAM International, ISBN 978-3-902734-05-1, ISSN 1726-9687, Vienna, Austria

Spencer, D. (2009). Card sorting: Designing usable categories. Brooklyn, NY: Rosenfeld Media. Tapscott, D. \& Tapscott, A. (2016). Blockchain revolution 2016, Available from: https://blockgeeks.com/guides/what-is-blockchain-technology/ Accessed: 2018-05-06 Teich, T.; Bodach, M.; Hoffman, M.; Neumann, T.; Werler, P. \& Hartel, T. (2017). Demographic Energy-balanced Framework for Sustainable Urban Environments, Proceedings of the 28th DAAAM International Symposium, pp.1044-1050, B. Katalinic (Ed.), Published by DAAAM International, ISBN 978-3-902734-11-2, ISSN 1726-9679, Vienna,

Austria

Wolfson, R. (2018). How A Leading Cyber Security Company Uses Blockchain Technology To Prevent Data Tampering, Forbes, Available from: https://www.forbes.com/sites/rachelwolfson/2018/07/03/how-a-leading-cybersecurity-company-uses-blockchain-technology-to-prevent-datatampering/\#3708c1bc4529 Accessed: 2018-08-15 *** Wikipedia, https://en.wikipedia.org/wiki/Blockchain Accessed: 2017-10-01 *** Wikipedia, https://en.wikipedia.org/wiki/Electric_utility Accessed: 2017-10-01 